\title{
High Definition X-Ray Fluorescence: Principles and Techniques
}

\author{
Z. W. Chen, Walter M. Gibson, and Huapeng Huang \\ X-Ray Optical Systems, Inc., 15 Tech Valley Drive, East Greenbush, NY 12061, USA \\ Correspondence should be addressed to Z. W. Chen, zchen@xos.com
}

Received 28 December 2007; Accepted 10 March 2008

Recommended by Ladislav Pina

Energy dispersive X-ray fluorescence (EDXRF) is a well-established and powerful tool for nondestructive elemental analysis of virtually any material. It is widely used for environmental, industrial, pharmaceutical, forensic, and scientific research applications to measure the concentration of elemental constituents or contaminants. The fluorescing atoms can be excited by energetic electrons, ions, or photons. A particular EDXRF method, monochromatic microfocus X-ray fluorescence (M $\mu \mathrm{EDXRF),} \mathrm{has} \mathrm{proven}$ to be remarkably powerful in measurement of trace element concentrations and distributions in a large variety of important medical, environmental, and industrial applications. When used with state-of-the-art doubly curved crystal (DCC) X-ray optics, this technique enables high-sensitivity, compact, low-power, safe, reliable, and rugged analyzers for insitu, online measurements in industrial process, clinical, and field settings. This new optic-enabled M $\mu$ EDXRF technique, called high definition X-ray fluorescence (HD XRF), is described in this paper.

Copyright (C) 2008 Z. W. Chen et al. This is an open access article distributed under the Creative Commons Attribution License, which permits unrestricted use, distribution, and reproduction in any medium, provided the original work is properly cited.

\section{Introduction}

X-ray fluorescence is a well-established and powerful tool for nondestructive elemental analysis of virtually any material. It is widely used for environmental, industrial, pharmaceutical, forensic, and scientific research applications to determine the presence or absence and in some cases to measure the concentration of elemental constituents or contaminants. The fluorescing atoms can be excited by energetic electrons, ions, or photons. The fluorescent X-rays are in general measured by two types of detection systems: wavelength dispersive detection (WDXRF) and energy dispersive detection (EDXRF). In the former, the fluorescence X-rays are diffracted from a single crystal or multilayer optic to select a particular and often very narrow wavelength (or X-ray energy) range, often corresponding to characteristic X-rays from an element of interest. This provides high elemental specificity and, because of the high signal-to-background ratio, often high sensitivity. EDXRF permits detection of a broad range of elements simultaneously. The measurement resolution (elemental specificity) depends in this case on the detector X-ray energy resolution and counting rate performance. A particular EDXRF method, monochromatic microfocus X-ray fluorescence ( $\mathrm{M} \mu \mathrm{EDXRF})$, has proven to be remarkably powerful in measurement of trace element concentrations and distributions for a large variety of important medical, environmental, and industrial applications [1]. In contrast with conventional X-ray fluorescence in which a broad spectrum of X-ray energies are incident on the sample, including the continuum Bremsstrahlung radiation resulting from electron scattering in the anode of a high-voltage $\mathrm{X}$-ray tube, excitation with monochromatic X-rays has greatly improved signal-to-noise ratio for measurement of secondary characteristic X-rays from the sample of interest. This is shown in Figure 1, which shows an XRF spectrum from an NIST SRM 2783 sample excited with a direct beam from a molybdenum X-ray tube and a spectrum from the same sample excited with a monochromatic beam from a doubly curved crystal (DCC) optic of the type described in this paper.

The Bremsstrahlung background in the conventional XRF spectrum is from scattering of source radiation in the sample. This can be avoided by selecting a characteristic $\mathrm{X}$-ray line from a target irradiated by the source to excite fluorescent X-rays in the sample. This secondary excitation technique is used in several commercial XRF systems [2] but requires a very high primary $\mathrm{X}$-ray source intensity to accommodate the large decrease in the X-ray intensity on 


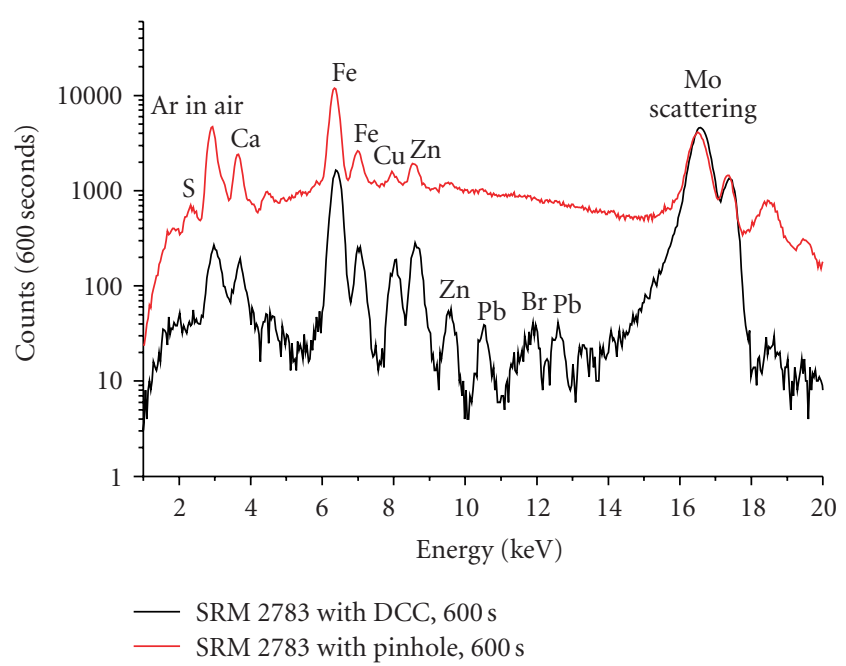

FIgURE 1: Comparison of XRF spectra taken from an NIST SRM 2783 standard reference sample using the monochromatic XRF method (black) and conventional XRF method (red).

the sample due to the secondary production process. Highenergy synchrotron sources are sometimes used to produce an intense beam of tunable monochromatic X-rays which are used for important material and medical XRF applications [3]. Another way to carry out X-ray fluorescence free of the Bremsstrahlung background is to use an ion beam to excite fluorescent X-rays in the sample of interest. This is the origin of the much studied particle-induced X-ray excitation (PIXE) technique [4]. All of these approaches illustrate the value of monochromatic excitation. However, they all require large, expensive, fixed, or laboratory-based facilities. Another approach to monochromatic X-ray fluorescence is the use of radioactive radiation sources such as ${ }^{109} \mathrm{Cd}$ and ${ }^{241} \mathrm{Am}$ [5]. Effective use of a ${ }^{241} \mathrm{Am}$ isotopic source provided elemental composition measurements on the famous Explorer Mars Lander and other planetary and lunar probes [6]. Terrestrial and even portable [7] analyzers utilizing radioactive sources have been used for environmental, industrial, and mineralogical applications but safety and regulatory considerations have constrained their widespread use. In addition, the need to keep the amount of radioactive material low often leads to reduced sensitivity and inconveniently large measurement time and sampling area. In addition, there is degradation and the ultimate need to replace the radioactive source.

The limitations of the techniques described above can be dramatically overcome by the use of state-of-the-art doubly curved crystal (DCC) X-ray optics which enable highsensitivity, compact, low-power, safe, reliable, and rugged analyzers for in-situ, online measurements in industrial process, clinical, and field settings $[8,9]$. In addition to the greatly improved signal-to-background ratio shown in Figure 1, it should be noted that the focused excitation beam from the DCC optic used in this example is more than 200 times smaller in area $(0.20 \mathrm{~mm}$ diameter $)$ than the $3 \mathrm{~mm}$ diameter beam needed for the direct excitation measurement to obtain the same incident beam flux as measured by the scattered Mo K $\alpha$ (elastic and inelastic) beam intensity. This new optic-enabled, monochromatic, microfocus, energy dispersive X-ray fluorescence (OEM $\mu \mathrm{EDXRF}$ ) technique is the subject of this paper and for brevity is called High Definition X-Ray Fluorescence (HD XRF).

\section{Doubly Curved Crystal (DCC) X-Ray Optics}

\subsection{Bragg Diffraction}

Doubly curved crystal (DCC) optics are based on the wellknown Bragg law $[10,11] n \lambda=2 d \sin \theta$, where $\lambda$ is the wavelength of the diffracted beam, $n$ is an integer indicting the order of diffraction from crystal planes of spacing $d$, and $\theta$ is the angle between the incident beam and the Bragg planes. For a highly collimated X-ray beam diffracted from a symmetric (with the diffracting planes parallel to the surface) perfect (flat) single crystal, the peak reflectivity reaches nearly unity over a small range of angles near the Bragg angle. From the dynamical theory [12], the range of angles over which this occurs is $\Omega=2 R \lambda^{2}\left(\sqrt{ } F_{h} F_{-h}\right) / \pi V \sin 2 \theta$, where $R$ is the classical electron radius $\left(2.818 \times 10^{-15} \mathrm{~m}\right), \lambda$ is the X-ray wavelength, the Fs are the structure factors for the reflections $(h k 1)$ and $(-h-k-1), V$ is the unit cell volume, and $\theta$ is the Bragg angle. This distribution can be modified by polarization effects ( 1 for $\sigma$ polarized X-rays and $\cos 2 \theta$ for $\pi$ polarized X-rays), and can be broadened (and reduced in intensity) by mosaic spread $w(\Delta)$ and crystal distortion effects. In addition, the peak reflectivity is reduced by absorption in the crystal which also makes the reflectivity curve asymmetric. Even small distortions can greatly perturb the behavior of perfect single crystals. Although there is no general theory that can handle arbitrary distortions, there are approximate treatments for special cases [13-15]. A summary of the intrinsic energy resolution for selected crystals and reflections for $1 \AA(12.4 \mathrm{keV}) \mathrm{X}$-rays is shown in Table I from Siddons [16]. The best possible energy and wavelength resolution is $\delta E / E=\delta \lambda / \lambda=\cot \theta \delta \theta+\delta \tau$, where $\delta E$ and $E$ are the energy band pass and the center energy of the beam, respectively, $\theta$ is the Bragg angle, and $\delta \tau$ is the intrinsic width of the crystal reflection as shown in Table 1 . The actual energy resolution is typically 2-3 times the calculated $\Delta E / E$ due to the effects discussed above.

\subsection{Curved Crystal Diffraction}

There are three reasons to apply a uniform curvature to a single crystal plate used as a monochromator: (1) to increase the diffracted beam intensity from a point X-ray source by meeting the Bragg condition over a larger range of incident angles from the divergent incident beam, (2) to improve the energy resolution for a divergent beam, and (3) to provide output beam concentration or focusing. For Bragg reflections, the most common geometry is based on the Roland circle principle which relies on the wellknown property of a circle that an arc segment subtends a constant angle for any point on the circle. In this geometry, the incidence angle for the local Bragg plane must be constant. The result of this was shown by Johansson [17] 
TABLE 1: Selected monochromator crystals with selected $d$-spacings and reflection widths for $1 \AA$ ( $12.4 \mathrm{keV}) \mathrm{X}$-rays.

\begin{tabular}{lcccc}
\hline Crystal & Reflection & $d$-spacing $(\mathrm{nm})$ & Refl. width $(\mu \mathrm{rad})$ & Intrinsic resolution, $\delta \tau$ \\
\hline Silicon & $(111)$ & 0.31355 & 22.3 & $1.36 \times 10^{-4}$ \\
Germanium & $(220)$ & 0.19201 & 15.8 & $5,37 \times 10^{-5}$ \\
& $(111)$ & 0.32664 & 50.1 & $3.1 \times 10^{-4}$ \\
Diamond & $(220)$ & 0.20002 & 37.4 & $1.37 \times 10^{-4}$ \\
& $(111)$ & 0.20589 & 15.3 & $5.8 \times 10^{-5}$ \\
Graphite & $(400)$ & 0.089153 & 5.2 & $7.4 \times 10^{-6}$ \\
\end{tabular}

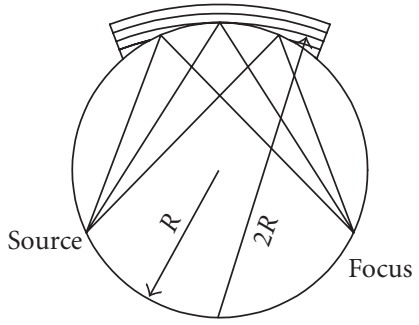

(a)

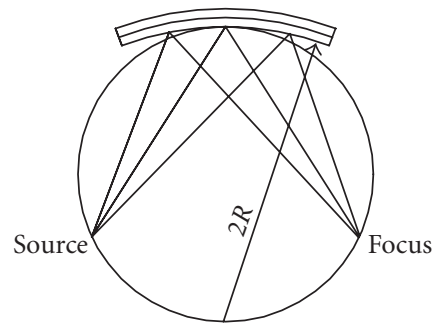

(b)

Figure 2: (a) The Johansson focusing monochromator, (b) the Johann focusing monochromator.

to require the radius of curvature to be twice the radius of the crystal surface. This can be achieved by a combination of bending and machining the crystal as shown in Figure 2(a). For applications requiring a small aperture, aberrations introduced by omitting the machining step are small and acceptable. This is the familiar Johann geometry [18] shown in Figure 2(b), which is the one used in the studies reported here.

Although the geometry shown in Figure 2 is symmetrical, it is possible to place the crystal anywhere on the circle and, therefore, achieve a (de)magnification of the source spot other than unity. In this case, the surface of the diffracting crystal must be cut at an angle to the Bragg planes in order to maintain the Bragg condition across the range of incidence angles. Depending on the source geometry, the bending radius, and symmetry of the crystal, the bending can improve or degrade the energy resolution of the monochromator. Each case must be considered in detail but it is possible to make some general observations. (1) If the angular aperture of the crystal as seen from the source is large enough to cover the intrinsic width (almost always true for perfect single crystals), the Roland circle geometry will improve the energy resolution. (2) When the absorption length becomes larger than the extinction distance for the X-rays inside the crystal, the X-rays can travel deeply into the bent crystal so that the incidence angle changes with depth leading to a broader energy band pass. (3) If the bending radius is small enough that the Bragg angle changes more than the intrinsic width, the peak reflectivity will decrease and the energy band pass will broaden. In practice, if the energy resolution is important, it should be measured experimentally.

\subsection{Doubly Curved Crystal Diffraction}

There is additional intensity benefit if the crystal is also curved in a plane perpendicular to the Roland circle as shown in Figure 3. The profile of the diffracted beam is shown in Figure 4. In the case of a toroidally curved crystal, the bending radius of the crystal perpendicular to the Roland circle is $R_{V}=2 R \sin ^{2} \theta_{B}$. This geometry gives point-to-point focusing on the Roland circle and the source spot $S$ is imaged at the image point $I$. As discussed below, other curvature geometries such as elliptical, logarithmic, and parabolic can be used to accommodate different source or image spot geometries. Toroidal curvature is frequently used in mirrors at synchrotron X-ray sources [19] which use small angle scattering rather than diffraction and is occasionally used in diffraction-based monochromators on synchrotrons where (because the curvature is facilitated by cutting grooves in the back of thick crystals) it is referred to as sagittal-focusing geometry.

Use of focusing geometries for producing a point image from a point X-ray source for analytical use was described and investigated during the 1950s [20, 21]. In the 1980 s and 1990s, the X-ray optical properties of DCC optics were systematically studied by using a ray tracing method [2226]. Fabrication and application of DCC optics were also reported in the early 1990s by Wittry et al. [27, 28]. However, widespread use and practical applications of DCC optics for monochromatic beam applications was impeded by the difficulty of fabricating DCC optics.

An important development was reported in 1997 and $1998[29,30]$. In these reports, an intense micro $\mathrm{Cu} \mathrm{K} \alpha 1$ 


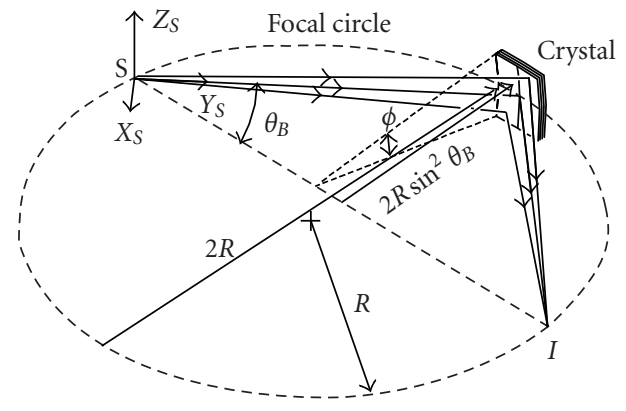

Bending radius in vertical

plane $=2 R \sin ^{2} \theta_{B}$

Bending radius in horizontal

plane $=2 R$

Axial symmetry along $S I$

FIGURE 3: Geometry of a toroidally curved doubly curved crystal (DCC) optic.

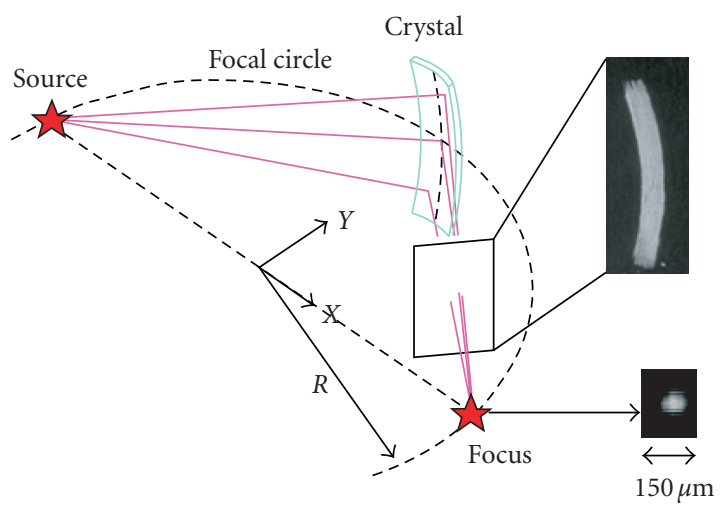

Figure 4: Beam profile at two points for toroidal DCC monochromator.

beam was obtained using a Johann-type doubly curved mica crystal for monochromatic microfocus XRF applications. The high intensity gain of the mica DCC was based on a novel crystal-bending technology. This technology can provide elastic bending of crystals into various complex shapes with precise figure control. The proprietary technology used has been refined and extended at X-Ray Optical Systems, Inc., (XOS) to produce the instruments and results summarized in the companion paper to this review [31].

Because of the large collection solid angle of DCC optics, intense monochromatic beams can be obtained even with low-power compact X-ray sources. Characteristics of selected monochromatic, focused, DCC-based X-ray beams are shown in Table 2.

A principal benefit of doubly curved crystal optics is the ability to provide an intense monochromatic focused beam. Various crystal materials can be used for DCC optics including Si, Ge, quartz, graphite, and mica. The collection solid angle of DCC optics is determined by the capture angle in the dispersive plane and the included rotational angle $\phi$.
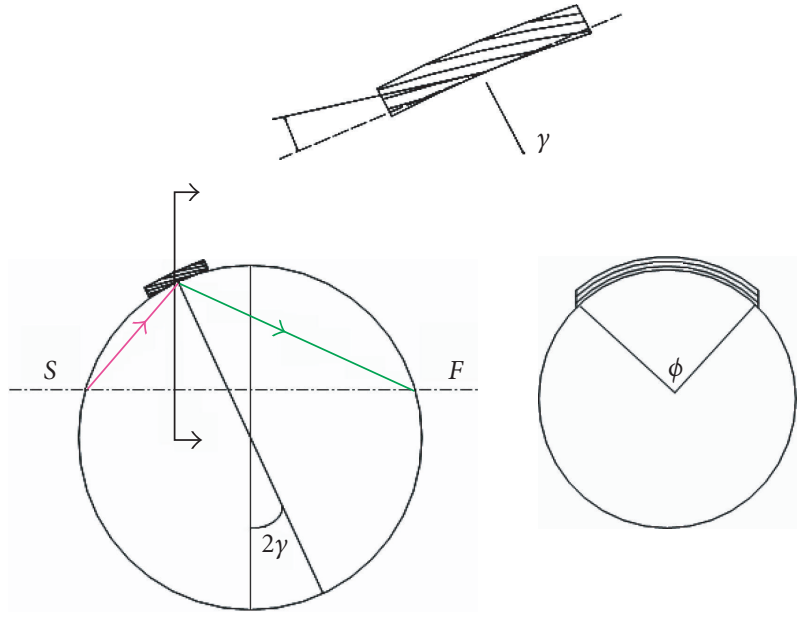

Figure 5: Asymmetric placement of DCC monochromator crystal for beam spot magnification.

Figures 3, 4, and Table 2 are for symmetrical toroidal optics. For such cases, the spot size is an image of the source.

In the Johan geometry, the optic can be placed nonsymmetrically on the Roland circle as shown in Figure 5. In this case, the crystal planes must be tilted with respect to the crystal surface an appropriate amount $\gamma$ depending on the displacement from the symmetry position. This can be used to demagnify the beam to obtain a smaller beam spot. Such demagnification has been used to produce a beam spot of $20 \mu \mathrm{m}$ diameter with a small spot Oxford Apogee source [32]. In the reverse mode, beam magnification can be achieved to obtain a weakly convergent beam while retaining a large input collection angle. This has been particularly useful for diffraction (XRD) studies which also benefit from the use of a monochromatic beam and can be especially useful for simultaneous XRD/XRF measurements for example in metallurgical and mineralogical (planetary) applications. In order to increase the monochromatic beam intensity, multiple diffracting crystals can be used as shown in Figure 6. The collection solid angle and, therefore, the beam intensity can also be increased by using noncircular profiles for the optic bending in the dispersive plane such as elliptical, parabolic, or inverse logarithmic spiral as shown in Figure 7. This is particularly useful at low X-ray energies where the Bragg diffraction angle is large and has been used with multiple tiled optic elements to obtain collection angles up to $30 \times 20$ degrees for sulfur $(2.3 \mathrm{keV}) \mathrm{X}$-rays [33]. For higher X-ray energies where the Bragg angle becomes small, the collection solid angle can be increased by the use of multiple optics wrapped around the included rotational angle $\phi$ as shown in Figures 8 and 9.

The capture angle in the dispersive plane is typically $1-$ 5 degrees and the rotational angle can be 5-300 degrees. The large potential collection efficiency in the rotational angle $\left(\sim 300^{\circ}\right)$ is made possible by the rotational symmetry around the S-I axis shown in Figure 2. The large solid angle of collection results in a monochromatic X-ray beam intensity produced with a low power $(20-50 \mathrm{~W}) \mathrm{X}$-ray source 
TABLE 2: Examples of nominal operating parameters for focusing, monochromatic X-ray beams integrated with microfocus X-ray sources.

\begin{tabular}{|c|c|c|c|c|c|c|c|}
\hline $\begin{array}{l}\text { Optic } \\
\text { crystal } \\
\text { X-ray } \\
\text { line }\end{array}$ & $\begin{array}{c}\text { Energy } \\
(\mathrm{keV})\end{array}$ & $\begin{array}{c}\text { Source } \\
\text { power } \\
(\mathrm{W})\end{array}$ & $\begin{array}{c}\text { Bragg } \\
\text { angle } \\
\left(\theta_{B}\right)\end{array}$ & $\begin{array}{c}\text { Capture } \\
\text { angle (sr) }\end{array}$ & $\begin{array}{c}\text { Nominal } \\
\text { focal spot } \\
\text { size, FWHM } \\
(\mu \mathrm{m})\end{array}$ & Flux (cps) & $\begin{array}{c}\text { Working } \\
\text { distance } \\
(\mathrm{mm})\end{array}$ \\
\hline \multicolumn{8}{|l|}{ Ge 220} \\
\hline $\mathrm{Cr} \mathrm{K} \alpha 1$ & 5.4 & 14 & 35.1 & 0.03 & 80 & $2 \times 10^{9}$ & 120 \\
\hline \multicolumn{8}{|l|}{ Si 111} \\
\hline $\mathrm{Cu} \mathrm{K} \alpha 1$ & 8.0 & 14 & 14.2 & 0.015 & 50 & $1 \times 10^{9}$ & 150 \\
\hline \multicolumn{8}{|l|}{ Si 111} \\
\hline $\mathrm{Cu} \mathrm{K} \alpha 1$ & 8.0 & 50 & 14.2 & 0.01 & 150 & $2 \times 10^{9}$ & 150 \\
\hline \multicolumn{8}{|l|}{ Si 220} \\
\hline $\mathrm{Cu} \mathrm{K} \alpha 1$ & 8.0 & 14 & 22.8 & 0.01 & 50 & $3 \times 10^{8}$ & 135 \\
\hline \multicolumn{8}{|l|}{ Si 220} \\
\hline W L $\alpha 1$ & 8.4 & 10 & 22.6 & 0.01 & 20 & $1 \times 10^{8}$ & 80 \\
\hline \multicolumn{8}{|l|}{ Si 220} \\
\hline Mo K $\alpha 1$ & 17.5 & 14 & 10.6 & 0.01 & 60 & $1 \times 10^{8}$ & 120 \\
\hline
\end{tabular}

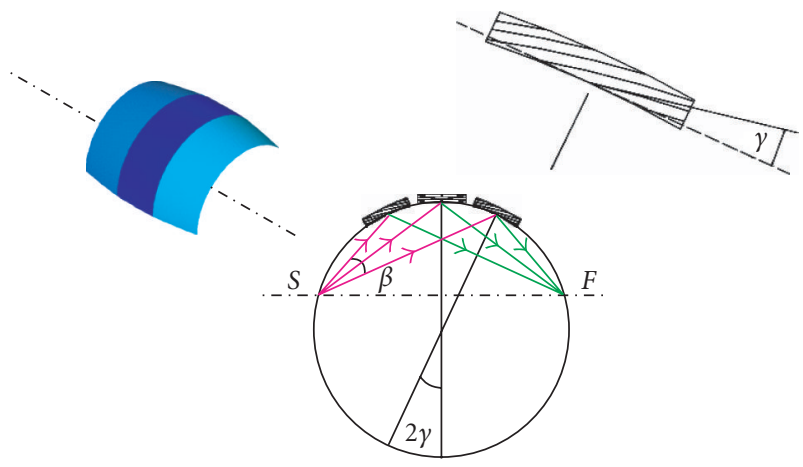

FIgURE 6: Use of multiple DCC crystals to increase focused beam intensity.

comparable to the intensity of a monochromatic beam obtainable from a high power $(5-10 \mathrm{~kW})$ rotating anode source using conventional optics. As noted above, the focal spot size of the diffracted beam is mainly determined by the $\mathrm{X}$-ray source size. The capabilities of commercially available DCC optics [34] are summarized in Table 3.

\section{X-Beam}

Efficient use of DCC optics requires a close match between the optic and the electron bombardment X-ray source spot on the anode. As noted in Section 2, for a well-made symmetrical toroidal optic, the source spot size is imaged at the optic focal point. For asymmetrical optics and for optic figures different from circular toroidal optics, a more complex relationship between the source spot size and the image focal spot size exists. For example, the focal spot for an inverse logarithmic spiral optic of the type shown in Figure 7 is much larger than the source spot size. For some applications this is acceptable, especially if the detector used has an input area larger than the spot size. It should be noted that it is not possible to reverse this optic in order to achieve a small spot size from a large source since $\mathrm{X}$-rays from each part of the source are incident on each part of the optic.

Virtually in every application, precise alignment of the optic with the source position is critical. This can often be a problem since the source spot position on the anode can vary as the source warms up or if the source power is changed, causing the alignment to change and the optic output intensity to vary. To alleviate this problem, proprietary Xbeams have been developed at XOS in which a thermal management system is used to keep the source spot constant during warm up of the source (which in some cases can take as long as two hours) or the source power is changed. In the X-beam assembly, the optic is prealigned and the desired beam intensity can be obtained within less than two or three minutes and held constant (usually within $0.3 \%$ ) for extended periods. Furthermore, the desired intensity can be reproduced through multiple on-off cycles of the X-ray system. In addition, X-Beams are internally shielded against radiation leakage and can contain an internal shutter and filter wheel assembly. Also, X-Beams with coupled DCC optics are air cooled, compact, and can be operated in any arbitrary orientation. A typical DCC X-Beam, about eight inches high and weighing less than five kilograms, is shown in the inset in Figure 9. This compact, low-power, safe, rugged, and reliable X-ray source-optic assembly is at the heart of most HD XRF analyzers discussed in the accompanying paper [31].

\section{Fundamental Parameters Analysis}

The ability to measure the intensity of X-ray fluorescence from individual elemental constituents in complex materials even when the amount present is low provides the possibility for rapid, nondestructive elemental analysis. As shown in 
TABLE 3: Characteristics of current DCC optics.

\begin{tabular}{ll}
\hline Output beam energies $(\mathrm{keV})$ & $\mathrm{Mo} \mathrm{L} \alpha 1,2.29 \mathrm{keV} ; \mathrm{Cr} \mathrm{K} \alpha 1,5.41 \mathrm{keV} ; \mathrm{Cu} \mathrm{K} \alpha 1,8.05 \mathrm{keV} ; \mathrm{WL} \beta 1,8.40 \mathrm{keV} ;$ \\
& $\mathrm{WL}_{\beta} 1,9.67 \mathrm{keV} ; \mathrm{Mo} \mathrm{K} \alpha 1,17.48 \mathrm{keV} ;$ or Ag K $\alpha 1,22.16 \mathrm{keV}$ \\
Reflection efficiency & $5 \%$ to $20 \%$ \\
Collection solid angle & 0.005 to 0.1 steradians \\
Convergent angles & $0.2-5$ degrees (dispersive plane) x 0.2-45 degrees (rotational plane) \\
Focused beam size & $20-300 \mu \mathrm{m}$ depending on the source size and optic design \\
\hline
\end{tabular}

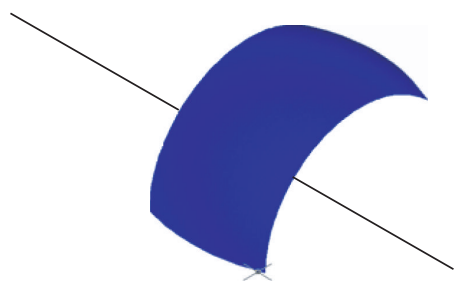

(a)

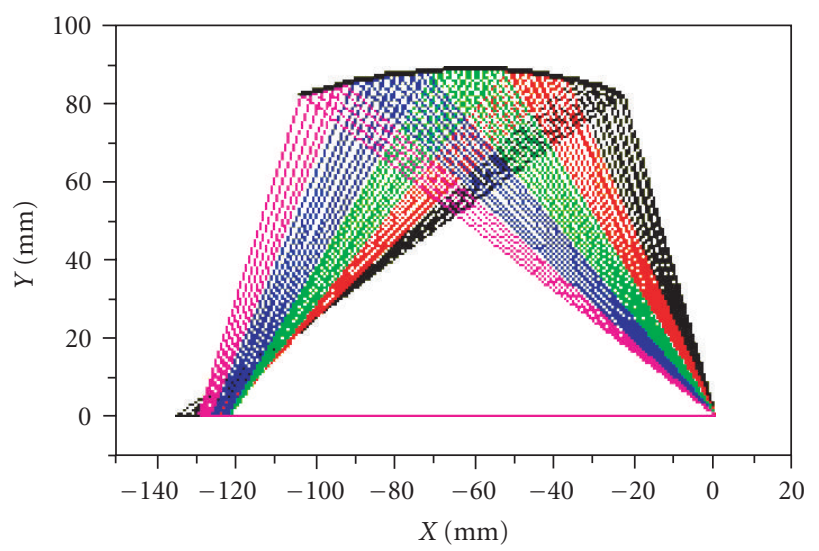

(b)

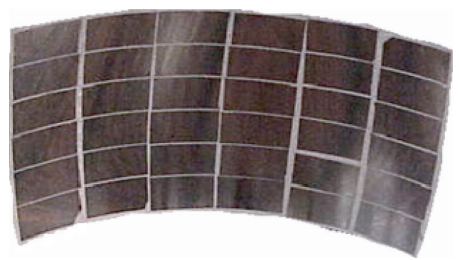

(c)

FIGURE 7: (a) Drawing of a revolving logspiral DCC optic for S K $\alpha$ (2.3 keV); (b) schematic of logarithmic curved DCC optic with Xrays ray from source on right to detector on left; (c) photograph of multisegment DCC optic used in SINDIE analyzer for Sulfur in diesel.

Figure 1, such measurements are greatly facilitated by the use of a monochromatic excitation beam. This has been recognized for decades. For very thin samples such as for air particulates collected on thin filters, quantitative analysis is straightforward. Such first principle calculations for thin samples require measurement of the integrated peak intensity for each element and application of experimentally

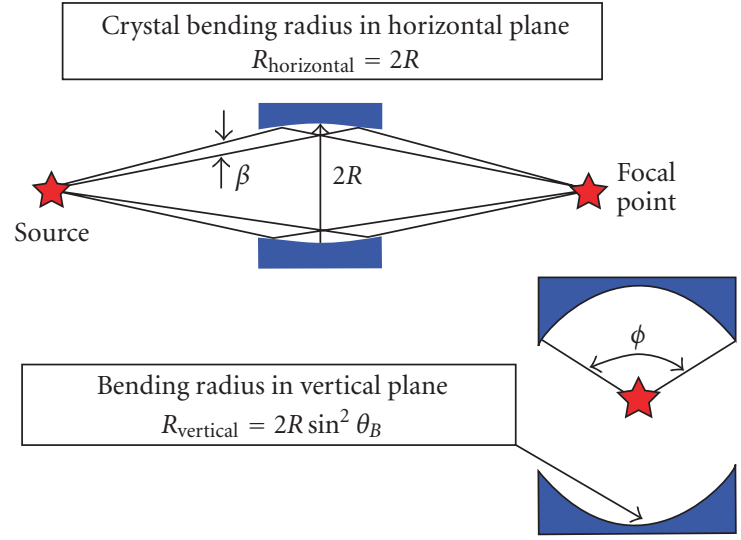

FIGURE 8: Schematic of rotationally wrap around DCC monochromators to increase focused beam intensity.

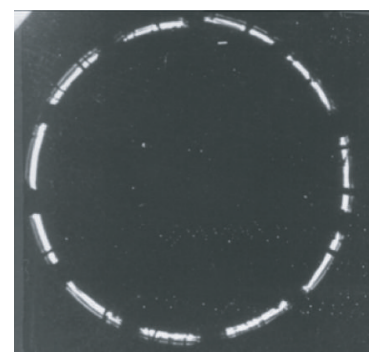

$$
\begin{aligned}
& R=646 \\
& r=19 \mathrm{~mm} \\
& \mathrm{IFD} / \mathrm{OFD}=2: 1 \\
& 80 \mu \text { spot }
\end{aligned}
$$

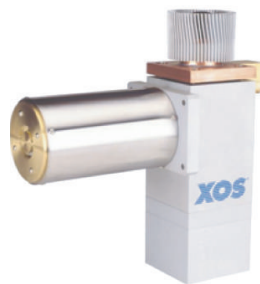

FIGURE 9: Beam profile from eleven-segment $\mathrm{Cu} \mathrm{K} \alpha$ rotationally wrap around demagnifying DCC optic with photograph of X-beam assembly (height of X-beam is 8 inches).

determined detector response functions and experimental [35] or calculated [36] fluorescent X-ray yields for pure samples of the elements of interest for a monochromatic excitation X-ray beam of the appropriate energy. The excitation probability is highest for elements with fluorescent $\mathrm{X}$-rays just below the energy of the exciting photons. An example of an experimental fluorescent X-ray excitation curve is shown in Figure 10 for a secondary $\mathrm{ZrK} \alpha_{1} \mathrm{~K} \beta$ (15.7$17.7 \mathrm{keV}$ ) excitation beam from Nielson [35]. For accurate measurement, it is still necessary in such measurements to make corrections for absorption and scattering from 


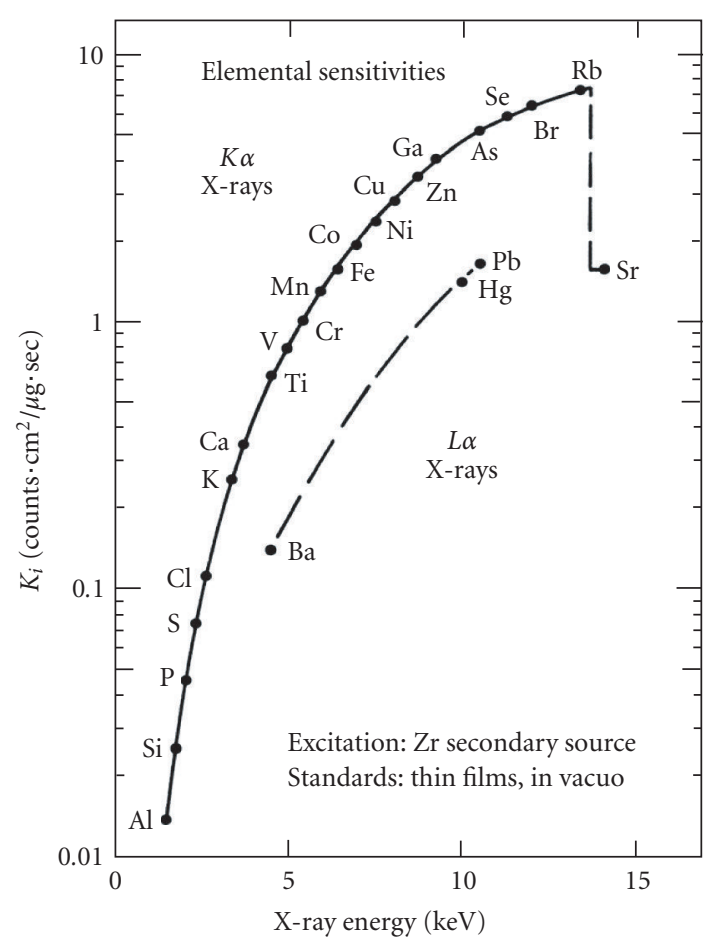

Figure 10: Experimental X-ray excitation curve measured for a $\mathrm{Zr}$ secondary source by the use of thin metal samples evaporated on thin Mylar substrate films from [35].

the measured elements and from the filter collector. Such corrections are more important for light elements such as sulfur but normally are still less than $10 \%$ of the uncorrected measurement [37]. For thicker samples, matrix scattering and absorption effects become much more important and suitable corrections and adjustment are crucial for quantitative application of X-ray fluorescence.

Since the seminal work of Compton [38], methods have been explored to make matrix corrections for X-ray fluorescence analysis. These have traditionally involved use of standards of known composition $[39,40]$ to calibrate the measurement system which must always take into account the detection method, geometry, and sample form. For conventional X-ray fluorescence, the analysis is complicated by the presence of a large Bremsstrahlung continuum which also reduces measurement sensitivity and requires background evaluation methods $[41,42]$. As noted in Section 1, introduction of monochromatic X-ray excitation dramatically improves the signal-to-background (S/B) ratio for trace element determinations by eliminating the background due to scattering in the sample of Bremsstrahlung radiation from the X-ray source. The clean, background-free X-ray spectrum also permits analysis of the ratio of the Rayleigh (coherent) and Compton scattering (incoherent) peaks for evaluation of X-ray absorption and scattering due to elements in the sample matrix. This is particularly important in evaluating the matrix effects from light elements (such as $\mathrm{H}, \mathrm{C}, \mathrm{N}$, and $\mathrm{O}$ ) that are not measured in the XRF spectrum. A comprehensive review of studies based on the use of the Compton scattering, especially for monochromatic (secondary and radioactive) sources, is given by Nielson [43]. A detailed analyses for matrix absorption and scattering effects for fundamental parameter (FP) analysis of a variety of materials from low $Z$ substrates such as petroleum to plant, mineral, and metal substrates using single ( $\mathrm{Zr})$ [44] and double ( $\mathrm{Zr}$ and $\mathrm{Ti}$ ) secondary $\mathrm{X}$-ray sources [45] is described by Nielsen using an iterative analysis procedure [35].

An HD XRF analysis software package built upon and extending the previous work is currently under development at XOS based on the fundamental parameter approach [46]. This fundamental parameter (FP) approach has already achieved notable success in analysis of thin, filter or impact membrane collected air particulate samples. As discussed in the accompanying paper, the use of HD XRF is particularly valuable for measurements of small particle fractions of importance for health studies and for the small sampling volumes and small sample sizes needed for portable personal environmental monitors. Measurement of ultratrace element concentrations in calibration samples prepared and characterized at the Trace Elements Laboratory at Wadsworth Center of the New York State Health Laboratory for evaluation of body fluids (urine, blood, and plasma) has shown excellent agreement with inductively coupled plasmamass spectroscopy (ICP-MS) characterized samples for lead and arsenic at concentrations from $10 \mathrm{ppb}$ to less than $50 \mathrm{ppb}$. These studies are part of an ongoing NIH funded study that has also shown the ability to analyze a variety of other elements $(\mathrm{Fe}, \mathrm{Cu}, \mathrm{Zn})$ of particular interest for studies of Alzheimer's Disease (AD) and other neurodegenerative disorders in collaboration with Alzheimer's Center at Albany Medical Center and the Wadsworth Center in Albany, NY, USA. A separate NIH funded study of trace metal deposits in autopsied brain and spinal cord tissues by use of HD XRF is also underway. Examples from these FP HD XRF studies as well as other studies are discussed in the accompanying paper [31].

Computer-based simulations are very useful to understand the X-ray scattering and absorption processes and the role of detector response functions and measurement geometries that lead to monochromatic EDXRF spectra for a variety of analyte elements over a wide range of concentrations in different sample matrices and thicknesses. This has been abundantly demonstrated in an extensive systematic program based on a Monte Carlo-Library LeastSquares method developed by Gardener et al. at North Carolina State University over more than two decades [4753]. A detailed summary of this method is given by Li et al. [54].

A very efficient analytical simulation method for HD XRF measurements is under development [46]. An example of such a simulation for a polyethylene standard sample containing controlled amounts of $\mathrm{Cr}, \mathrm{Hg}, \mathrm{Pb}$, and $\mathrm{Br}$ is shown in Figure 11. This simulation is being refined to more completely account for secondary scattering effects. Analyses of thick plastic standards are shown in Table 4 with concentrations of observed heavy element constituents obtained from fitting of simulated peak intensities as shown 
TABLE 4: Reference and measured values for three polyethylene standards. The experimental uncertainty (at 95\% level) includes estimated systematic uncertainty plus Poisson counting statistical uncertainty.

\begin{tabular}{lcccccc}
\hline & & $\mathrm{Cr}$ & $\mathrm{Hg}$ & $\mathrm{Pb}$ & $\mathrm{Br}$ & Uncertainty \\
\hline PE_5398_N & Ref. & 1001 & 1000 & 1000 & 500 & $4 \%(95 \%$ level) \\
& Mea. & 1020 & 950 & 981 & 442 & $10 \%(95 \%$ level) \\
PE_H_18A & Ref. & 1000 & 1100 & 1200 & 1100 & $4 \%(95 \%$ level) \\
& Mea. & 1020 & 1150 & 1220 & 1090 & $10 \%(95 \%$ level) \\
PE_L & Ref. & 400 & 200 & 400 & 500 & $4 \%(95 \%$ level) \\
& Mea. & 401 & 185 & 419 & 508 & $10 \%(95 \%$ level) \\
\hline
\end{tabular}

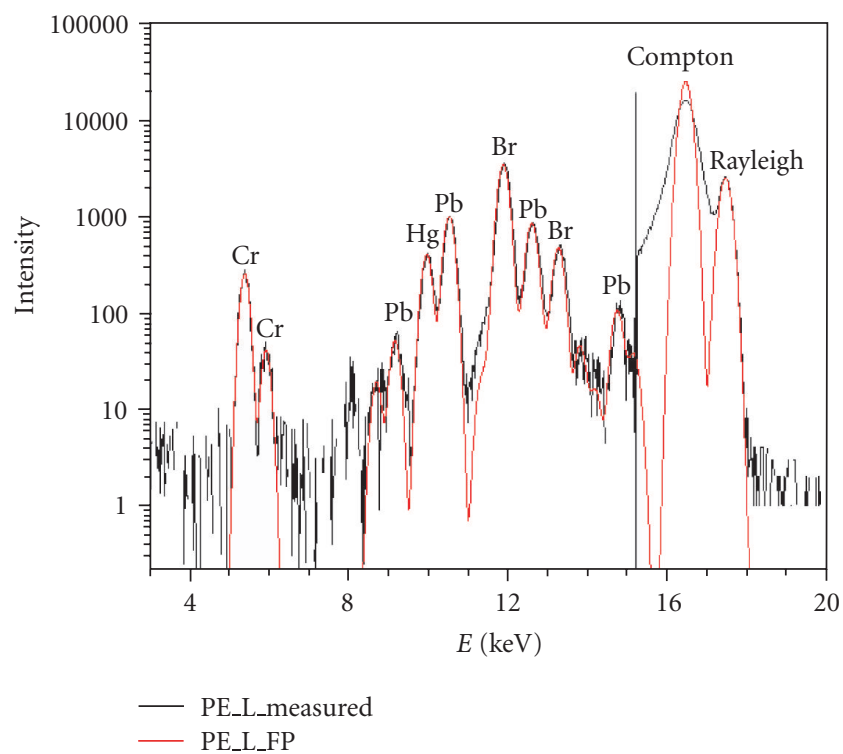

FIGURE 11: HD XRF spectrum from polyethylene standard PE 5398 L sample and analytical simulated spectrum from fundamental parameters analysis. A background subtraction was made for the spectrum below $15.2 \mathrm{keV}$.

in Figure 11. Even without further refinement, agreement with the reference values is very close and provides a basis for reverse FP analyses for thin or thick water or plasticmatrix homogeneous samples. The analysis is currently being refined for other plastic, organic, or metallic homogeneous matrices.

The physical constants used in the FP analysis are the incident and exit angle, the energy of the incident monochromatic X-ray beam, tabulated mass attenuation coefficients [55], fluorescence yields [56], absorption jump ratios [57], intensity ratios of lines within a given series (e.g., $\mathrm{K} \alpha / \mathrm{K} \beta$ ratio), and energies of absorption edges and emission lines [58].

The effects of particle size in inhomogeneous matrices and samples with layers of differing materials and constituents are under examination. A special but important class of layered inhomogeneous samples is paint on a thick homogeneous substrate. Both experimental and analytical studies are underway for FP analysis of such cases. Pre-

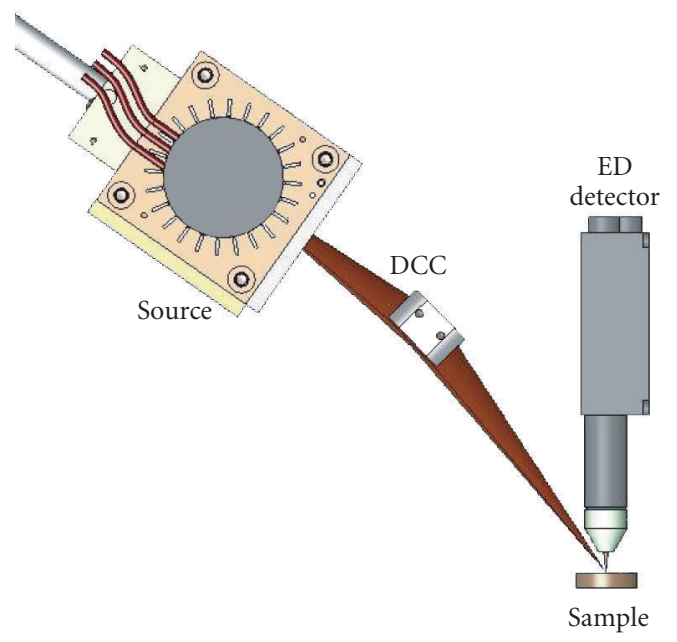

FIGURE 12: Schematic of laboratory benchtop HD XRF analyzer.

liminary results show promise for such applications and are discussed in the accompanying paper on HD XRF applications [31].

\section{Conclusions}

A new HD XRF system based on the use of proprietary DCC $\mathrm{X}$-ray optics coupled with low-power X-ray tube sources in a proprietary, compact, portable, safe, stable, X-beam assembly for monochromatic, microfocus, energy dispersive, $\mathrm{X}$-ray fluorescence enables a broad variety of in situ, online, clinical, or remote applications in science, medicine, and industry. The principles and techniques as well as the basis for automated fundamental parameter (FP) (standardless) analysis that is enabled by this new analytical tool are discussed in this report. Selected applications are reviewed in an accompanying paper [31]. A schematic diagram of a laboratory-based HD XRF system of the type used for the measurement shown in Figure 11 and Table 4 is shown in Figure 12. Development of specialized HD XRF analyzers based on this approach is discussed in the companion paper [31]. 


\section{Acknowledgments}

Development of HD XRF has been supported by a number of Phase I and Phase II SBIR grants from several federal agencies: DOE, DE-FG-02-99ER82918; NIH, 2 R44 RR1493502; NSF, DMI-0091570; NIH, DMI-0215914; NIH, 2 R44 CA88678-02; DOE, DE-FG02-04ER84122; EPA, PR-NC-0410308. The content is solely the responsibility of the authors and does not necessarily represent the official views of the Department of Energy, the National Institutes of Health, the National Science Foundation, or the Environmental Protection Agency. The authors would also like to acknowledge discussions and active participation with colleagues at X-Ray Optical Systems, Inc., and at the Center for X-Ray Optics at the University at Albany, State University of New York.

\section{References}

[1] R. Jenkins, An Introduction to X-Ray Spectrometry, Hayden and Son, New York, NY, USA, 1974.

[2] J. M. Jaklevic and R. D. Giauque, "Semiconductor detectors for X-ray spectrometry," in Handbook of X-Ray Spectrometry, R. E. Van Grieken and A. A. Markowicz, Eds., p. 170, Marcel Dekker, New York, NY, USA, 1993.

[3] K. W. Jones, "Synchrotron radiation induced X-ray emission," in Handbook of X-Ray Spectrometry, R. E. Van Grieken and A. A. Markowicz, Eds., p. 411, Marcel Dekker, New York, NY, USA, 1993.

[4] W. Maenhauf and K. G. Malmqvistk, "Particle-induced X-ray emission analysis," in Handbook of X-Ray Spectrometry, R. E. Van Grieken and A. A. Markowicz, Eds., p. 517, Marcel Dekker, New York, NY, USA, 1993.

[5] J. S. Watt, "Radioisotopte X-ray analysis," in Handbook of XRay Spectrometry, R. E. Van Grieken and A. A. Markowicz, Eds., p. 389, Marcel Dekker, New York, NY, USA, 1993.

[6] B. C. Clark and A. B. Baird, "Ultraminiaturize X-ray fluorescence spectrometer for in-situ geochemical analysis on Mars," Earth and Planetary Science Letters, vol. 19, no. 3, pp. 359-368, 1973.

[7] Portable (handheld) radioactive source XRF analyzer Ref., http://www.rmd-leadtracer.com/RoHS/.

[8] N. Mail, C. A. MacDonald, Z. Chen, and W. M. Gibson, "Potential application of doubly curved crystal optics," in XRay Sources and Optics, vol. 5537 of Proceedings of SPIE, pp. 94-104, Denver, Colo, USA, August 2004.

[9] W. M. Gibson, Z. Chen, N. Gao, H. Huang, and T. C. Miller, "X-ray optics, an enabling technology for science, medicine, and industry," in Laser-Generated, Synchrotron, and Other Laboratory X-Ray and EUV Sources, Optics, and Applications II, vol. 5918 of Proceedings of SPIE, pp. 1-18, San Diego, Calif, USA, August 2005.

[10] W. H. Bragg and W. L. Bragg, "The reflection of X-rays by crystals," Proceedings of the Royal Society of London A, vol. 88, no. 605 , pp. 428-438, 1913.

[11] W. H. Bragg and W. L. Bragg, "The reflection of X-rays by crystals-II," Proceedings of the Royal Society of London A, vol. 89, no. 610, pp. 246-248, 1913.

[12] W. H. Zachariasen, Theory of X-Ray Diffraction in Crystals, Dover, New York, NY, USA, 1967.

[13] D. Taupin, "Théorie dynamique de la diffraction des rayons $\mathrm{X}$ par les cristaux déformés," Bulletin de la Société Française de Minéralogie et de Cristallographie, vol. 87, pp. 469-511, 1964.
[14] S. Takagi, "Dynamical theory of diffraction applicable to crystals with any kind of small distortion," Acta Crystallographica, vol. 15, no. 12, pp. 1311-1312, 1962.

[15] P. Penning, D. Polder, et al., "Anomalous transmission of Xrays in ellastically deformed crystals," Philps Research Report, vol. 16, pp. 419-440, 1961.

[16] P. Siddons, Handbook of Optics, Vol. III, chapter 22, McGrawHill, New York, NY, USA, 2001.

[17] T. Johansson, "Über ein neuartiges, genau fokussierendes Röntgenspektrometer," Zeitschrift für Physik A, vol. 82, no. 78, pp. 507-528, 1933.

[18] H. H. Johann, "Die Erzeugung lichtstarker Röntgenspektren mit Hilfe von Konkavkristallen," Zeitschrift für Physik A, vol. 69, no. 3-4, pp. 185-206, 1931.

[19] A. Freund, Handbook of Optics, Vol III, chapter 26, McGrawHill, New York, NY, USA, 2001.

[20] G. Hagg and N. Karlsson, "Aluminum monochromator with double curvature for high-intensity X-ray powder photographs," Acta Crystallographica, vol. 5, part 6, pp. 728-730, 1952.

[21] D. W. Berreman, J. W. M. DuMond, and P. E. Marmier, "New point-focusing monochromator," Review of Scientific Instruments, vol. 25, no. 12, pp. 1219-1220, 1954.

[22] D. B. Wittry and D. M. Golijanin, "Alignment and characterization of doubly-curved X-ray diffractors," in Microbeam Analysis, pp. 51-55, San Francisco Press, San Francisco, Calif, USA, 1987.

[23] D. B. Wittry and S. Sun, "X-ray optics of doubly curved diffractors," Journal of Applied Physics, vol. 67, no. 4, pp. 16331638, 1990.

[24] D. B. Wittry and S. Sun, "Focusing properties of curved X-ray diffractors," Journal of Applied Physics, vol. 68, no. 2, pp. 387391, 1990.

[25] D. B. Wittry and S. Sun, "Properties of curved X-ray diffractors with stepped surfaces," Journal of Applied Physics, vol. 69, no. 7, pp. 3886-3892, 1991.

[26] D. B. Wittry and S. Sun, "X-ray optics of doubly curved diffractors-II," Journal of Applied Physics, vol. 71, no. 2, pp. 564-568, 1992.

[27] D. B. Wittry, "Focussing X-rays for microprobe X-ray fluorescence analysis," in Proceedings of the 50th Annual Meeting of the Electron Microscopy Society of America, MAS 27th Annual Meeting and MSC/SMC 19th Annual Meeting, vol. , pp. 17301731, San Francisco Press, San Francisco, Calif, USA, 1992.

[28] D. B. Wittry, W. Z. Chang, and R. Y. Li, "New developments in curved diffractor geometries for X-ray microanalysis," Microbeam Analysis, vol. 2, pp. S192-S193, 1993.

[29] Z. W. Chen and D. B. Wittry, "Microprobe X-ray fluorescence with the use of point-focusing diffractors," Applied Physics Letters, vol. 71, no. 13, pp. 1884-1886, 1997.

[30] Z. W. Chen and D. B. Wittry, "Microanalysis by monochromatic microprobe X-ray fluorescence-physical basis, properties, and future prospects," Journal of Applied Physics, vol. 84, no. 2, pp. 1064-1073, 1998.

[31] W. M. Gibson, Z. Chen, and D. Li, "High definition Xray diffraction: applications," Journal of X-Ray Optics and Instruments. In press.

[32] Z. Chen and W. M. Gibson, "Doubly curved crystal (DCC) Xray optics and applications," Powder Diffraction, vol. 17, no. 2, pp. 99-103, 2002.

[33] Z. W. Chen, F. Wei, I. Radley, and B. Beumer, "Low-level Sulfur in fuel determination using monchromatic WD XRF-ASTM D 7039-04," Journal of ASTM International, vol. 2, no. 8, pp. 49-53, 2005. 
[34] X-ray Optical Systems, Inc., 15 Tech Valley Rd. East Greenbush, NY, USA.

[35] K. K. Nielson, "Matrix corrections for energy dispersive $\mathrm{X}$-ray fluorescence analysis of environmental samples with coherent/incoherent scattered X-rays," Analytical Chemistry, vol. 49, no. 4, pp. 641-648, 1977.

[36] W. H. McMaster, N. K. Del Grande, J. H. Mallett, and J. H. Hubbell, "Compilation of X-ray cross sections," Tech. Rep. UCRL 50174, Section II, Revision I, Lawrence Radiation Laboratory, University of California, Berkeley, Calif, USA, 1969.

[37] K. K. Nielson and S. R. Garcia, "Use of X-ray scattering in absorption corrections for X-ray fluorescence analysis of aerosol loaded filters," Advances in X-ray Analysis, vol. 20, pp. 497-506, 1977, Proceedings of the 25th Annual Conference on Applications of X-ray Analysis.

[38] A. H. Compton and S. K. Allison, X-rays in Theory and Experiment, Van Nostrand, New York, NY, USA, 1935.

[39] R. D. Giauque, F. S. Goulding, J. M. Jaklevic, and R. H. Pehl, "Trace element determination with semiconductor detector X-ray spectrometers," Analytical Chemistry, vol. 45, no. 4, pp. 671-681, 1973.

[40] R. D. Giauque, R. B. Garrett, and L. Y. Goda, "Energy dispersive X-ray fluorescence spectrometry for determination of twenty-six trace and two major elements in geochemical specimens," Analytical Chemistry, vol. 49, no. 1, pp. 62-67, 1977.

[41] P. van Espen and F. Adams, "Evaluation of a practical background calculation method in X-ray energy analysis," $X$ Ray Spectrometry, vol. 5, no. 3, pp. 123-128, 1976.

[42] C. E. Feather and J. P. Willis, "A simple method for background and matrix correction of spectral peaks in trace element determination by X-ray fluorescence spectrometry," X-ray Spectrometry, vol. 5, no. 1, pp. 41-48, 1976.

[43] K. K. Nielson, "Progress in X-ray fluorescence correction methods using scattered radiation," Advances in X-Ray Analysis, vol. 22, pp. 303-315, 1979.

[44] K. K. Nielson and R. W. Sanders, "Multielement analysis of unweighed biological and geological samples using backscatter and fundamental parameters," Advances in X-ray Analysis, vol. 26, pp. 385-390, 1983.

[45] R. W. Sanders, K. B. Olsen, W. C. Weimer, and K. K. Nielson, "Multielement analysis of unweighed oil samples by X-ray fluorescence spectroscopy with two excitation sources," Analytical Chemistry, vol. 56, no. 12, pp. 1911-1914, 1983.

[46] H. Huang and Z. Chen, private communication.

[47] A. R. Hawthorne and R. P. Gardner, "Monte Carlo models for the inverse calculation of multelement amounts in XRF analysis," Transactions of the American Nuclear Society, vol. 21, supplement 3, pp. 38-39, 1975.

[48] A. R. Hawthorne and R. P. Gardner, "Monte Carlo simulation of X-ray fluorescence from homogeneous multielement samples excited by continuous and discrete energy photons from X-ray tubes," Analytical Chemistry, vol. 47, no. 13, pp. 2220 2225, 1975.

[49] F. Arinc, L. Wielopolski, and R. P. Gardner, "The linear least-squares analysis of X-ray fluorescence spectra of aerosol samples using pure element library standards and photon excitation," in X-Ray Fluorescence Analysis of Environmental Samples, T. J. Dzubay, Ed., pp. 227-240, Ann Arbor Science, Ann Arbor, Mich, USA, 1977.

[50] R. P. Gardner, A. Sood, Y. Y. Wang, L. Liu, P. Guo, and R. J. Gehrke, "Single peak versus library least-squares analysis methods for the PGNAA analysis of vitrified waste," Applied
Radiation and Isotopes, vol. 48, no. 10-12, pp. 1331-1335, 1997.

[51] W. Guo, R. P. Gardner, and A. C. Todd, "Using the Monte Carlo-Library Least-Squares (MCLLS) approach for the in vivo XRF measurement of lead in bone," Nuclear Instruments and Methods in Physics Research Section A, vol. 516, no. 2-3, pp. 586-593, 2004.

[52] T. He, R. P. Gardner, and K. Verghese, "The Monte CarloLibrary Least-Squares approach for energy-dispersive X-ray fluorescence analysis," Applied Radiation and Isotopes, vol. 44, no. 10-11, pp. 1381-1388, 1993.

[53] S. H. Lee, R. P. Gardner, and A. C. Todd, "Preliminary studies on combining the $\mathrm{K}$ and $\mathrm{L}$ XRF methods for in vivo bone lead measurement," Applied Radiation and Isotopes, vol. 54, no. 6, pp. 893-904, 2001.

[54] F. Li, W. Guo, and R. P. Gardner, "Implementation of the Monte Carlo-Library least-squares method to energy dispersive X-ray fluorescence analysis".

[55] W. J. Veigele, "Mass attenuation and absorption cross sections for 94 elements; $0.1 \mathrm{keV}$ to $1 \mathrm{MeV}$," in Handbook of Spectroscopy, J. W. Robinson, Ed., vol. 1, p. 28, CRC Press, Cleveland, Ohio, USA, 1974.

[56] W. Bambynek, B. Crasemann, R. W. Fink, et al., "X-ray fluorescence yields, auger, and coster-kronig transition probabilities," Reviews of Modern Physics, vol. 44, no. 4, pp. 716-813, 1972.

[57] P. V. Rao, M. H. Chen, and B. Crasemann, "Atomic vacancy distributions produced by inner-shell ionization," Physical Review A, vol. 5, no. 3, pp. 997-1012, 1972.

[58] J. A. Bearden, "X-ray wavelengths," Reviews of Modern Physics, vol. 39, no. 1, pp. 78-124, 1967. 